\title{
Instantaneous and noninstantaneous dissolution: approximation by the finite volume method
}

\author{
R. Eymard, T. Gallouët, R. Herbin, D. Hilhorst, M. Mainguy
}

Résumé. Nous étudions des schémas de volumes finis pour l'approximation de la solution d'équations non linéaires de diffusion-dissolution. Nous montrons la convergence du schéma, ainsi que la convergence de la solution du problème continu vers la solution du problème limite de la dissolution instantanée. Un cas d'application, portant sur une expérience de lixiviation du béton, est présenté.

Mots clés. Diffusion-dissolution, volumes finis, limite singulière, dissolution instantanée.

\begin{abstract}
This paper is devoted to the study of finite volume schemes for nonlinear dissolution-diffusion equations. The approximate solution is shown to converge to the continuous one, which is also shown to converge in the limit of instantaneous dissolution. The scheme is applied to the interpretation of an experiment of leaching of cement concrete.
\end{abstract}

Keywords. Diffusion-dissolution, finite volumes, singular limit, instantaneous dissolution

AMS Subject Classification. 35L05, 35T

\section{Introduction.}

In several countries one plans to store radioactive waste in deep disposals. The efficiency of such disposals relies on material barriers. For such a use, cement concrete offers the advantage of having a weak porosity. Nevertheless, disposal safety relies on the durability of concrete, subjected to the agression of water, which dissolves the calcium included in the skeletton mineral constituants (the leaching phenomenon).

It is essential to be able to forecaste the evolution of the porosity and the permeability of the concrete, since it is shown that these properties increase with calcium leaching. Simple models of this phenomenon can be drawn, in order to evaluate its importance. In such models, only one mineral specie is considered as solvable in the liquid phase, with a linear kinetics. Then the dissolved mineral molecules diffuse in the liquid phase, yielding 
a displacement of mineral mass towards the boundaries of the material. The limit problem in the case of instantaneous diffusion turns out to be a free boundary problem, since the domain is separated into two regions: a first one in which all the solvable mineral molecules are dissolved and in which the concentration in the liquid phase is equal to zero, and a second one in which there remains some unsolved mineral molecules in the solid phase, and the concentration in the liquid phase is close to the saturation concentration. It is of interest to study the influence of the kinetics of dissolution compared to the kinetics of diffusion which is slowed down by the tortuosity of the porous medium. We do so in this paper by seeking numerical approximations of the continuous solutions, and by comparing the mathematical behaviour of schemes in both the cases of instantaneous and noninstantaneous dissolution. This study also yields a complete understanding of the singular behavior of the continuous solution in the limit of instantaneous dissolution.

In order to perform the numerical approximation, we apply the finite volume method which has proven to be robust and simple when approximating nonlinear conservation laws. It is well-known that respecting the local conservation of species is one of the easiest ways to ensure the correct location of the fronts, in both the cases of instantaneous and noninstantaneous dissolution.

We suppose that the following hypotheses are satisfied :

(i) $\Omega$ is a bounded open subset of $\mathbb{R}^{N}$, with smooth boundary $\partial \Omega$,

(ii) $U$ and $V$ are given positive constants,

(iii) $u_{0} \in L^{\infty}(\Omega)$ satisfies $0 \leq u_{0} \leq U$,

(iv) $v_{0} \in L^{\infty}(\Omega)$ satisfies $0 \leq v_{0} \leq V$,

(v) $\lambda$ is a given positive constant.

Our purpose is to find a numerical approximation of solutions of the system

$$
\begin{cases}(u+v)_{t}-\Delta u & =0 \\ v_{t} & = \begin{cases}-\lambda(U-u) & \text { if } v>0 \\ 0 & \text { if } v=0\end{cases} \end{cases}
$$

which modelizes dissolution-diffusion, and of solutions of the system

$$
\begin{cases}(u+v)_{t}-\Delta u & =0 \\ v \cdot(U-u) & =0, \text { with } v \geq 0, u \leq U\end{cases}
$$

which modelizes instantaneous dissolution-diffusion.

We assume that $u$ satisfies the homogeneous Neumann boundary condition

$$
\frac{\partial u}{\partial n}=0, \quad \text { on } \partial \Omega \times \mathbb{R}_{+}^{*},
$$

and that $u$ and $v$ satisfy the initial conditions 


$$
\left\{\begin{array}{l}
u(x, 0)=u_{0}(x) \\
v(x, 0)=v_{0}(x)
\end{array}\right.
$$

for $x \in \Omega$.

In what follows we denote by $\left(P_{\lambda}\right)$ the problem $(2,4,5)$ and by $\left(P_{L}\right)$ the problem $(3$, $4,5)$. Problem $\left(P_{\lambda}\right)$ represents a phenomenon of dissolution-diffusion in a porous medium $\Omega \subset \mathbb{R}^{N}$. The function $u$ represents the concentration of a mineral constituant dissolved in the static liquid phase of the porous medium, and $v$ the concentration of the same mineral constituant in the solid phase. The dissolved constituant can diffuse in the liquid phase, and the kinetics of the dissolution reaction is given by a first order law which does not hold in the case of precipitation. In this law, the positive constant $\lambda$ stands for the inverse of a dissolution time, and the positive constant $U$ is the equilibrium concentration at saturation. Problem $\left(P_{L}\right)$ corresponds to the case of instantaneous dissolution.

We now give a definition of a weak solution $\left(u_{\lambda}, v_{\lambda}\right)$ of Problem $\left(P_{\lambda}\right)$. We use the notation $a^{+}:=\max (a, 0)$.

Definition 1.1 A pair of measurable functions $\left(u_{\lambda}, v_{\lambda}\right)$ is a weak solution of Problem $\left(P_{\lambda}\right)$ if for all $T>0$

$$
\left\{\begin{array}{l}
u_{\lambda} \in L^{\infty}(\Omega \times(0, T)) \cap L^{2}\left(0, T ; H^{1}(\Omega)\right), v_{\lambda} \in L^{\infty}(\Omega \times(0, T)), \\
\int_{0}^{T} \int_{\Omega}\left(\left[u_{\lambda}(x, t)+v_{\lambda}(x, t)\right] \psi_{t}(x, t)-\nabla u_{\lambda}(x, t) \nabla \psi(x, t)\right) d x d t+ \\
\int_{\Omega}\left[u_{0}(x)+v_{0}(x)\right] \psi(x, 0) d x=0, \\
\text { for all } \psi \in \mathcal{A}_{T}=\left\{\psi \in H^{1}(\Omega \times(0, T)) \text { with } \psi(., T)=0\right\} \\
v_{\lambda}(x, t)=\left(v_{0}(x)-\lambda \int_{0}^{t}\left(U-u_{\lambda}(x, \tau)\right) d \tau\right)^{+}, \text {for a.e. }(x, t) \in \Omega \times(0, T) .
\end{array}\right.
$$

Remark 1.2 In (6), test functions cannot be taken in the set $\left\{\psi \in L^{2}\left(0, T ; H^{1}(\Omega)\right), \psi_{t} \in L^{2}\left(0, T ; H^{-1}(\Omega)\right)\right.$ with $\left.\psi(., T)=0\right\}$ since $v_{0}$ only belongs to $L^{\infty}(\Omega)$.

The existence and uniqueness of the weak solution of Problem $\left(P_{\lambda}\right)$ follows from $[10]$ and [9].

Definition 1.3 A pair of measurable functions $(u, v)$ is a weak solution of Problem $\left(P_{L}\right)$ if for all $T>0$ 


$$
\left\{\begin{array}{l}
u \in L^{\infty}(\Omega \times(0, T)) \cap L^{2}\left(0, T ; H^{1}(\Omega)\right), v \in L^{\infty}(\Omega \times(0, T)), \\
\int_{0}^{T} \int_{\Omega}\left([u(x, t)+v(x, t)] \psi_{t}(x, t)-\nabla u(x, t) \nabla \psi(x, t)\right) d x d t+ \\
\int_{\Omega}\left[u_{0}(x)+v_{0}(x)\right] \psi(x, 0) d x=0, \\
\text { for all } \psi \in \mathcal{A}_{T}=\left\{\psi \in H^{1}(\Omega \times(0, T)) \text { with } \psi(., T)=0\right\} \\
v \cdot(U-u)=0 \text { with } v \geq 0, \quad u \leq U \text { a.e. in } \Omega \times(0, T) .
\end{array}\right.
$$

For a weak solution $(u, v)$ of Problem $\left(P_{L}\right)$, we set $w=u+v$. The condition $v \cdot(U-u)=0$ with $v \geq 0$ and $u \leq U$ a.e. in $\Omega \times(0, T)$ implies that, introducing the function $\varphi(s):=\min (s, U)$, the equalities $u=\varphi(w)$ and $v=\max (0, w-U)$ hold a.e. in $\Omega \times(0, T)$. Hence we get from ( 7$)$ that, for all $T>0$, the function $w$ is a solution of the following equation:

$$
\left\{\begin{array}{l}
w \in L^{\infty}(\Omega \times(0, T)), \varphi(w) \in L^{2}\left(0, T ; H^{1}(\Omega)\right), \\
\int_{0}^{T} \int_{\Omega}\left(w(x, t) \psi_{t}(x, t)-\nabla \varphi(w)(x, t) \nabla \psi(x, t)\right) d x d t+ \\
\int_{\Omega}\left[u_{0}(x)+v_{0}(x)\right] \psi(x, 0) d x=0, \\
\text { for all } \psi \in \mathcal{A}_{T}=\left\{\psi \in H^{1}(\Omega \times(0, T)) \text { with } \psi(., T)=0\right\} .
\end{array}\right.
$$

Equation (8) defines a weak solution of a one-phase Stefan problem with the homogeneous Neumann boundary condition and initial value $\left(u_{0}+v_{0}\right)$. The existence and uniqueness of such a function $w$ is a classical result, presented in [7] in a more general context. Since any solution $(u, v)$ of Problem $\left(P_{L}\right)$ satisfies $u=\varphi(w)$ and $v=\max (0, w-U)$, we deduce the existence and uniqueness of the solution of Problem $\left(P_{L}\right)$.

The organization of this paper is as follows: in Section 2 we define a finite volume scheme for the diffusion-dissolution Problem $\left(P_{\lambda}\right)$. The same scheme is also used for solving the instantaneous diffusion-dissolution Problem $\left(P_{L}\right)$. In Section 3 we present a priori estimates for the approximate solution $\left(u_{\mathcal{T}, k}, v_{\mathcal{T}, k}\right)$ of Problem $\left(P_{\lambda}\right)$ : first $L^{\infty}$ estimates for $u_{\mathcal{T}, k}$ and $v_{\mathcal{T}, k}$ and then estimates on differences of space and time translates of $u_{\mathcal{T}, k}$. It is remarkable that these estimates are uniform as well in the discretization steps as in the inverse of the dissolution time $\lambda$ and yield a convergence proof of the discrete solution to the weak solution of Problem $\left(P_{\lambda}\right)$. The convergence of the weak solution of Problem $\left(P_{\lambda}\right)$ to that of Problem $\left(P_{L}\right)$ is proven in Section 4. Finally we present a realistic numerical test in Section 5. 


\section{Finite volume scheme for dissolution-diffusion equations.}

In this section, we define approximate solutions of the Problems $\left(P_{\lambda}\right)$ and $\left(P_{L}\right)$. To this purpose, we introduce a finite volume space discretization and a time discretization using Euler's scheme.

\subsection{Finite volume space discretization.}

Definition 2.1 (admissible finite volume mesh of $\Omega$ )

$\mathcal{T}$ is an admissible finite volume mesh of $\Omega$ if and only if :

(i) $\mathcal{T}$ is a finite set of disjoint open subsets of $\Omega$ such that $\bigcup_{K \in \mathcal{T}} \bar{K}=\bar{\Omega}$,

(ii) the elements of $\mathcal{T}$ will be called control volumes in what follows. For all $K \in \mathcal{T}$, we denote by $m(K)$ its measure for the Lebesgue measure of $\mathbb{R}^{N}$. For any $(K, L) \in \mathcal{T}^{2}$ with $K \neq L$, we denote by $e_{K, L}=\bar{K} \cap \bar{L}$ their common interface, which is supposed to be included in a hyperplane of $\mathbb{R}^{N}$, which does not intersect $K$ nor $L$. Then $m\left(e_{K, L}\right)$ denotes the measure of $e_{K, L}$ for the Lebesgue measure of the hyperplane, and $\mathbf{n}_{K, L}$ denotes the unit vector normal to $e_{K, L}$, oriented from $K$ to $L$. The set of pairs of adjacent control volumes is denoted by $\mathcal{E}=\left\{(K, L) \in \mathcal{T}^{2}, K \neq L, m\left(e_{K, L}\right) \neq 0\right\}$, and for all $K \in \mathcal{T}$, $N(K)=\{L \in \mathcal{T},(K, L) \in \mathcal{E}\}$ denotes the set of neighbours of $K$,

(iii) there exist $x_{K} \in K$, for all $K \in \mathcal{T}$, such that :

$$
\frac{x_{L}-x_{K}}{\left|x_{L}-x_{K}\right|}=\mathbf{n}_{K, L}, \text { for all }(K, L) \in \mathcal{E} \text {. }
$$

We denote by $h=\operatorname{size}(\mathcal{T})$ the positive number $h=\sup _{K \in \mathcal{T}} \delta(K)$, where $\delta(K)$ denotes the diameter of control volume $K$. Moreover, $d_{K, L}=\left|x_{L}-x_{K}\right|$ is the euclidian distance between the points $x_{K}$ and $x_{L}$, and we then set $\tau_{K, L}=\frac{m\left(e_{K, L}\right)}{d_{K, L}}$.

Let $\mathcal{T}$ be an admissible mesh and $k>0$ a time step. The explicit finite volume scheme is defined by the following equations.

The initial condition for the scheme is given by

$$
\text { for all } K \in \mathcal{T}\left\{\begin{aligned}
u_{K}^{0} & =\frac{1}{m(K)} \int_{K} u_{0}(x) d x \\
v_{K}^{0} & =\frac{1}{m(K)} \int_{K} v_{0}(x) d x \\
w_{K}^{0} & =u_{K}^{0}+v_{K}^{0} .
\end{aligned}\right.
$$

The explicit finite volume scheme for Problem $\left(P_{\lambda}\right)$ is defined by

$$
\left\{\begin{array}{l}
m(K)\left[\frac{w_{K}^{n+1}-w_{K}^{n}}{k}\right]-\sum_{L \in N(K)} \tau_{K, L}\left(u_{L}^{n}-u_{K}^{n}\right)=0 \\
\text { for all } K \in \mathcal{T}, \text { for all } n \in \mathbb{N},
\end{array}\right.
$$


which yields a unique value $w_{K}^{n+1}$, for all $K \in \mathcal{T}$ and for all $n \in \mathbb{N}$, and

$$
\begin{cases}v_{K}^{n+1}-\left(v_{K}^{n}-k \lambda\left(U-w_{K}^{n+1}+v_{K}^{n+1}\right)\right)^{+} & =0, \\ u_{K}^{n+1}+v_{K}^{n+1} & =w_{K}^{n+1}, \\ \text { for all } K \in \mathcal{T}, \text { for all } n \in \mathbb{N} . & \end{cases}
$$

Note that since the left hand side of the first equation of (11) is a strictly increasing continuous function of $v_{K}^{n+1}$, the equations (11) lead to a unique pair $\left(u_{K}^{n+1}, v_{K}^{n+1}\right)$. These values satisfy

$$
v_{K}^{n+1}=\left(v_{K}^{n}-k \lambda\left(U-u_{K}^{n+1}\right)\right)^{+} .
$$

If we take the limit $\lambda \rightarrow \infty$ in the first equation of (11), we obtain

$$
\left\{\begin{array}{l}
v_{K}^{n+1}=\left(w_{K}^{n+1}-U\right)^{+}, \\
u_{K}^{n+1}+v_{K}^{n+1} \\
\text { for all } K \in \mathcal{T}, \text { for all } n \in \mathbb{N},
\end{array}=w_{K}^{n+1},\right.
$$

which constitutes, together with $(10)$ a numerical scheme for Problem $\left(P_{L}\right)$, since it follows from (13) that $u_{K}^{n+1}=\min \left(w_{K}^{n+1}, U\right)$. The convergence of this scheme has been proved in [8]. One possibility would have been to study simultaneously the convergence of both schemes, since we shall see in the following that the a priori estimates do not depend on $\lambda$. However, we prefer to show the convergence of the finite volume scheme in the case of non instantaneous dissolution, after which we prove the convergence of the weak solution of Problem $\left(P_{\lambda}\right)$ to that of Problem $\left(P_{L}\right)$ as $\lambda \longrightarrow \infty$.

Equation (10) formally corresponds to integrating the first of the equations (2) on the element $K \times(n k,(n+1) k)$ and defining a suitable explicit approximation of the flux function across $\partial K$. An implicit approximation is also possible, and the existence of a solution of the resulting nonlinear system of equations can be easily proven by a fixed point method [9]. The study of the explicit scheme is slightly more difficult because of the conditions on the time step. Equation (11) corresponds to a time integration of the second equation of (2) and a projection on the set of non negative functions. The numerical scheme $(9,10,11)$ allows to build approximate solutions of Problem $\left(P_{\lambda}\right)$, denoted by $u_{\mathcal{T}, k}: \Omega \times \mathbb{R}^{+} \mapsto \mathbb{R}$ and $v_{\mathcal{T}, k}: \Omega \times \mathbb{R}^{+} \mapsto \mathbb{R}^{+}$by

$$
\left\{\begin{array}{l}
u_{\mathcal{T}, k}(x, t)=u_{K}^{n} \\
v_{\mathcal{T}, k}(x, t)=v_{K}^{n}
\end{array}\right.
$$

for all $x \in K$ and for all $t \in[n k,(n+1) k)$.

\section{A priori estimates.}

\section{1 $L^{\infty}$-estimate on $u, L^{2}$-estimate on $v$.}


Lemma 3.1 Suppose that hypotheses (1) are satisfied and let $T>0$ be given. Let $\mathcal{T}$ be an admissible mesh in the sense of Definition 2.1 and $k>0$ be a given time step. Assume that the condition

$$
k \leq \frac{m(K)}{\sum_{L \in N(K)} \tau_{K, L}}, \quad \text { for all } K \in \mathcal{T},
$$

is satisfied. Then, for all $n \in \mathbb{N}$, the sequence $\left(u_{K}^{n}\right)_{K \in \mathcal{T}}$ defined by $(9,10,11)$ is such that

$$
0 \leq u_{K}^{n} \leq U \text { for all } K \in \mathcal{T}, \text { for all } n \in \mathbb{N},
$$

which implies in particular that the function $u_{\mathcal{T}, k}$ defined by (14) satisfies

$$
\left\|u_{\mathcal{T}, k}\right\|_{L^{\infty}(\Omega \times(0, T))} \leq U
$$

Proof By hypothesis (1.iii), (16) is true for $n=0$. Next we suppose that it is satisfied up to step $n$ and prove that it also holds at step $n+1$. Let $K \in \mathcal{T}, n \in \mathbb{N}$. We deduce from (12) that there exists $\alpha_{K}^{n} \in[0,1]$ such that,

$$
\frac{v_{K}^{n+1}-v_{K}^{n}}{k}=\alpha_{K}^{n} \lambda\left(u_{K}^{n+1}-U\right) .
$$

With this equation, the first equation of the scheme (10) can be rewritten as :

$$
\begin{aligned}
u_{K}^{n+1}\left(1+k \lambda \alpha_{K}^{n}\right)= & u_{K}^{n}\left(1-\frac{k}{m(K)} \sum_{L \in N(K)} \tau_{K, L}\right)+ \\
& \frac{k}{m(K)} \sum_{L \in N(K)} \tau_{K, L} u_{L}^{n}+k \lambda \alpha_{K}^{n} U .
\end{aligned}
$$

We deduce from (15) that the quantity in factor of $u_{K}^{n}$ in (19) is always nonnegative. In view of (16) the expression of $v_{K}^{n+1}$ in (11) shows that for all $K \in \mathcal{T}$ the sequence $\left(v_{K}^{n}\right)_{n \in \mathbb{N}}$ is nonincreasing. Some easy consequences are the following ones.

Lemma 3.2 Suppose that hypotheses (1) are satisfied and let $T>0$ be given. Let $\mathcal{T}$ be an admissible mesh in the sense of Definition 2.1 and $k>0$ be a given time step such that condition (15) is satisfied.

Then, for all $n \in \mathbb{N}$, the sequence $\left(v_{K}^{n}\right)_{K \in \mathcal{T}}$ defined by $(9,10,11)$ is such that

$$
0 \leq v_{K}^{n+1} \leq v_{K}^{n} \leq v_{K}^{0} \text { for all } K \in \mathcal{T}, \text { for all } n \in \mathbb{N},
$$

and therefore

$$
\sum_{n \in \mathbb{N}} \sum_{K \in \mathcal{T}} m(K)\left|v_{K}^{n+1}-v_{K}^{n}\right| \leq\left\|v_{0}\right\|_{L^{2}(\Omega)} \sqrt{m(\Omega)}
$$


Hence the function $v_{\mathcal{T}, k}$ defined by (14) satisfies

$$
\left\|v_{\mathcal{T}, k}\right\|_{L^{2}(\Omega \times(0, T))} \leq \sqrt{T}\left\|v_{0}\right\|_{L^{2}(\Omega)}
$$

\subsection{Estimate of the space translations of approximate solutions $\left(u_{\mathcal{T}, k}\right)$.}

Lemma 3.3 Suppose that hypotheses (1) are satisfied and let $T>0$ be given. Let $\mathcal{T}$ be an admissible mesh in the sense of Definition 2.1 and $k$ be a given time step with $0<k<T$. Let $\alpha \in(0,1)$ be given and assume that the condition

$$
k \leq(1-\alpha) \frac{m(K)}{\sum_{L \in N(K)} \tau_{K, L}}, \text { for all } K \in \mathcal{T},
$$

is satisfied. Then there exists $F_{1}>0$, which only depends on $\Omega, U, u_{0}$ and $v_{0}$ such that the sequence $\left(u_{K}^{n}\right)_{K \in \mathcal{T}, n \in \mathbb{N}}$ defined by $(9,10,11)$ verifies

$$
\sum_{n=0}^{[T / k]} k \sum_{(K, L) \in \mathcal{E}} \tau_{K, L}\left(u_{K}^{n}-u_{L}^{n}\right)^{2} \leq \frac{F_{1}}{\alpha},
$$

where $[T / k]=\max \{n \in N ; n k \leq T\}$.

Note that the condition (23) is stronger than (15). Therefore, the result of Lemma 3.1 holds, i.e. $0 \leq u_{K}^{n} \leq U$, for all $K \in \mathcal{T}, n=0, \ldots[T / k]$. The proof of Lemma 3.3 can be done by following the steps of the continuous estimates. The following lemma, concerning space translates, is an essential consequence of the previous one, and is the first step which will make it possible to apply Kolmogorov's theorem (the second step on time translates being presented below).

Lemma 3.4 Under the hypotheses of Lemma 3.3, there exists $F_{1}>0$, which only depends on $\Omega, U, u_{0}$ and $v_{0}$ such that the function $u_{\mathcal{T}, k}$ defined by $(9,10,11)$ and (14) verifies

$$
\int_{\Omega_{\xi} \times(0, T)}\left(u_{\mathcal{T}, k}(x+\xi, t)-u_{\mathcal{T}, k}(x, t)\right)^{2} d x d t \leq|\xi|(|\xi|+2 h) \frac{F_{1}}{\alpha},
$$

for all $\xi \in \mathbb{R}^{N}$, where $\Omega_{\xi}=\{x \in \Omega,[x+\xi, x] \subset \Omega\}$.

The proof of Lemma 3.4 is presented in [7].

\subsection{Estimate of the time translations of approximate solutions $\left(u_{\mathcal{T}, k}\right)$.}

Lemma 3.5 Under the hypotheses of Lemma 3.3, there exist $F_{1}>0$ and $F_{2}>0$, which only depend on $\Omega, U, u_{0}$ and $v_{0}$ such that the function $u_{\mathcal{T}, k}$ defined by $(9,10,11)$ and $(14)$ satisfies 


$$
\int_{\Omega \times(0, T-\tau)}\left(u_{\mathcal{T}, k}(x, t+\tau)-u_{\mathcal{T}, k}(x, t)\right)^{2} d x d t \leq \tau\left(2 \frac{F_{1}}{\alpha}+F_{2}\right),
$$

for all $\tau \in(0, T)$.

It is possible to show compactness properties without Lemma 3.5, using for example the semi-group method [4]. Nevertheless, this theory only applies to implicit schemes. We can now apply the following lemma which is a consequence of Kolmogorov's theorem (cf. [7]). It permits to obtain a strong convergence property in $L^{2}(\Omega \times(0, T))$ from the estimates (25) and (26).

Lemma 3.6 Let $\left(f_{m}\right)_{m \in \mathbb{N}}$ be a sequence of functions of $L^{2}(\Omega \times(0, T))$ which verifies

1. there exists $M_{1}>0$ such that for all $m \in \mathbb{N},\left\|f_{m}\right\|_{L^{\infty}(\Omega \times(0, T))} \leq M_{1}$,

2. there exists $M_{2}>0$ such that for all $m \in \mathbb{N}$ and $\tau \in(0, T)$,

$$
\int_{\Omega \times(0, T-\tau)}\left(f_{m}(x, t+\tau)-f_{m}(x, t)\right)^{2} d x d t \leq \tau M_{2}
$$

3. there exist $M_{3}>0$ and a sequence of real positive values $\left(h_{m}\right)_{m \in \mathbb{N}}$ with $\lim _{m \rightarrow \infty} h_{m}=0$ such that for all $m \in \mathbb{N}$ and $\xi \in \mathbb{R}^{N}$,

$$
\int_{\Omega_{\xi} \times(0, T)}\left(f_{m}(x+\xi, t)-f_{m}(x, t)\right)^{2} d x d t \leq|\xi|\left(|\xi|+h_{m}\right) M_{3},
$$

where $\Omega_{\xi}=\{x \in \Omega,[x+\xi, x] \subset \Omega\}$.

Then there exists a subsequence of $\left(f_{m}\right)_{m \in \mathbb{N}}$ which converges to an element of $L^{2}\left(0, T ; H^{1}(\Omega)\right)$ in the strong topology of $L^{2}(\Omega \times(0, T))$.

\subsection{Convergence of the scheme}

The above results permit to prove the following convergence result, since they yield the existence of convergent subsequences whose limit can be proved to be solution of Problem $\left(P_{\lambda}\right)$ (the proof is given in $[9]$ ).

Theorem 3.7 Suppose that hypotheses (1) are satisfied, let $T>0$ and $\alpha \in(0,1)$ be given. Then for all $\varepsilon>0$, there exists $h_{0}>0$ such that for all $(\mathcal{T}, k)$ satisfying

1. $\mathcal{T}$ is an admissible mesh in the sense of Definition 2.1 and $h=\operatorname{size}(\mathcal{T}) \leq h_{0}$,

2. $k$ is a time step with $0<k<T$ satisfying the condition (23) for the mesh $\mathcal{T}$,

3. $u_{\mathcal{T}, k}$ and $v_{\mathcal{T}, k}$ are given by $(9,10,11)$ and $(14)$, for the mesh $\mathcal{T}$ and the time step $k$, 
the following inequalities hold

$$
\left\{\begin{array}{l}
\left\|u_{\mathcal{T}, k}-u_{\lambda}\right\|_{L^{2}(\Omega \times(0, T))} \leq \varepsilon \\
\left\|v_{\mathcal{T}, k}-v_{\lambda}\right\|_{L^{2}(\Omega \times(0, T))} \leq \varepsilon
\end{array}\right.
$$

i.e. $\left(u_{\mathcal{T}, k}\right)$ and $\left(v_{\mathcal{T}, k}\right)$ converge to the weak solution $\left(u_{\lambda}, v_{\lambda}\right)$ of Problem $\left(P_{\lambda}\right)$ as size $(\mathcal{T}) \rightarrow$ 0 . Moreover, there exist positive functions $F_{1}>0$ and $F_{2}>0$ of $\Omega, U, u_{0}$ and $v_{0}$ such that

$$
\left\|u_{\lambda}\right\|_{L^{2}\left(0, T ; H^{1}(\Omega)\right)} \leq \sqrt{\frac{F_{1}}{\alpha}}
$$

and

$$
\int_{\Omega \times(0, T-\tau)}\left(u_{\lambda}(x, t+\tau)-u_{\lambda}(x, t)\right)^{2} d x d t \leq \tau\left(2 \frac{F_{1}}{\alpha}+F_{2}\right),
$$

for all $\tau \in(0, T)$, and

$$
\left\|v_{\lambda}\right\|_{L^{\infty}(\Omega \times(0, T))} \leq\left\|v_{0}\right\|_{L^{\infty}(\Omega)}
$$

\section{Limit of the weak solution of problem $\left(P_{\lambda}\right)$ as $\lambda \rightarrow \infty$}

In this section, we use the fact that the estimates obtained for the approximate solutions of Problem $\left(P_{\lambda}\right)$ do not depend on $\lambda$. They yield the estimates (28), (29) and (30) for the weak solution $\left(u_{\lambda}, v_{\lambda}\right)$ of Problem $\left(P_{\lambda}\right)$ which also do not depend on $\lambda$. These estimates are used to prove the convergence of $\left(u_{\lambda}, v_{\lambda}\right)$ to the weak solution $(u, v)$ of Problem $\left(P_{L}\right)$ as $\lambda \rightarrow \infty$.

Theorem 4.1 Let $\Omega$ be an open bounded set of $\mathbb{R}^{N}$ with smooth boundary and let $T>0$. We suppose that $u_{0} \in L^{\infty}(\Omega), 0 \leq u_{0} \leq U$ where $U>0$ is a positive constant, and that $v_{0} \in L^{\infty}(\Omega)$ is a positive function. Let $(u, v)$ be the unique weak solution of Problem $\left(P_{L}\right)$ and, for all $\lambda>0$, let $\left(u_{\lambda}, v_{\lambda}\right)$ be the weak solution of problem $\left(P_{\lambda}\right)$.

Then

(i) For all $\lambda>0,0 \leq u_{\lambda} \leq U$, and there exists a function $F>0$ of $\Omega, u_{0}, v_{0}$ and $U$ such that $\left\|u_{\lambda}\right\|_{L^{2}\left(0, T ; H^{1}(\Omega)\right)} \leq F$ and

$$
\int_{0}^{T-\tau} \int_{\Omega}\left(u_{\lambda}(x, t+\tau)-u_{\lambda}(x, t)\right)^{2} d x d t \leq F \tau, \text { for all } \tau \in(0, T),
$$

(ii) For all $\lambda>0,0 \leq v_{\lambda}(x, t) \leq v_{0}(x)$ for a.e. $(x, t) \in \Omega \times(0, T)$,

(iii) $u_{\lambda} \rightarrow u$ in $L^{2}(\Omega \times(0, T))$ as $\lambda \rightarrow+\infty, v_{\lambda} \rightarrow v$ for the weak star topology of $L^{\infty}(\Omega \times(0, T))$ as $\lambda \rightarrow+\infty$. In particular the function $u$ satisfies as well the inequalities $\|u\|_{L^{2}\left(0, T ; H^{1}(\Omega)\right)} \leq F$ and $\int_{0}^{T-\tau} \int_{\Omega}(u(x, t+\tau)-u(x, t))^{2} d x d t \leq F \tau$. 
Proof Items (i) and (ii) of the above theorem are direct consequences of the convergence of the finite volume scheme. Hence there exists a pair of functions $(\tilde{u}, \tilde{v}) \epsilon$ $L^{2}\left(0, T ; H^{1}(\Omega)\right) \times L^{\infty}(\Omega \times(0, T))$ with $0 \leq \tilde{u} \leq U, 0 \leq \tilde{v} \leq V$, and a subsequence of $\left(u_{\lambda}, v_{\lambda}\right)_{\lambda>0}$, which we denote again by $\left(u_{\lambda}, v_{\lambda}\right)_{\lambda>0}$, such that $u_{\lambda} \rightarrow \tilde{u}$ in $L^{2}(\Omega \times(0, T))$ and $v_{\lambda} \rightarrow \tilde{v}$ for the weak star topology of $L^{\infty}(\Omega \times(0, T))$ as $\lambda \rightarrow+\infty$. In particular the function $\tilde{u}$ satisfies as well the inequalities $\|\tilde{u}\|_{L^{2}\left(0, T ; H^{1}(\Omega)\right)} \leq F$ and $\int_{0}^{T-\tau} \int_{\Omega}(\tilde{u}(x, t+$ $\tau)-\tilde{u}(x, t))^{2} d x d t \leq F \tau$. In order to prove (iii), we have to prove that $(\tilde{u}, \tilde{v})$ is the weak solution of Problem $\left(P_{L}\right)$. Passing to the limit in Problem $\left(P_{\lambda}\right)$, we have that

$$
\left\{\begin{array}{l}
\tilde{u} \in L^{\infty}(\Omega \times(0, T)) \cap L^{2}\left(0, T ; H^{1}(\Omega)\right), \tilde{v} \in L^{\infty}(\Omega \times(0, T)), \\
\int_{0}^{T} \int_{\Omega}\left([\tilde{u}(x, t)+\tilde{v}(x, t)] \psi_{t}(x, t)-\nabla \tilde{u}(x, t) \nabla \psi(x, t)\right) d x d t+ \\
\int_{\Omega}\left[u_{0}(x)+v_{0}(x)\right] \psi(x, 0) d x=0, \\
\text { for all } \psi \in \mathcal{A}_{T}=\left\{\psi \in H^{1}(\Omega \times(0, T)) \text { with } \psi(., T)=0\right\}
\end{array}\right.
$$

It only remains to show that $\tilde{v} \cdot(U-\tilde{u})=0$ a.e. in $\Omega \times(0, T)$.

We set $z_{\lambda}(x, t)=\int_{0}^{t}\left(U-u_{\lambda}(x, \tau)\right) d \tau$. The function $z_{\lambda}$ converges strongly in $L^{2}(\Omega \times$ $(0, T))$ to $z(x, t)=\int_{0}^{t}(U-\tilde{u}(x, \tau)) d \tau$. We consider $A_{\lambda}=\int_{0}^{T} \int_{\Omega} z_{\lambda}(x, t) v_{\lambda}(x, t) d x d t$. On the one hand, since $v_{\lambda} \rightarrow \tilde{v}$ for the weak star topology of $L^{\infty}(\Omega \times(0, T))$ as $\lambda \rightarrow+\infty$, we deduce that $A_{\lambda} \rightarrow \int_{0}^{T} \int_{\Omega} z(x, t) \tilde{v}(x, t) d x d t$ as $\lambda \rightarrow+\infty$.

On the other hand, for a.e. points $(x, t) \in \Omega \times(0, T)$, either $z_{\lambda}(x, t) \leq \frac{v_{0}(x)}{\lambda}$ holds and then we have $z_{\lambda}(x, t) v_{\lambda}(x, t) \leq \frac{\left(v_{0}(x)\right)^{2}}{\lambda}$, or $z_{\lambda}(x, t) \leq \frac{v_{0}(x)}{\lambda}$ does not hold and $v_{\lambda}(x, t)=$ $\left(v_{0}(x)-\lambda z_{\lambda}(x, t)\right)^{+}=0$. Therefore $A_{\lambda} \leq \frac{T}{\lambda}\left\|v_{0}\right\|_{L^{2}(\Omega)}^{2}$, which tends to zero as $\lambda \rightarrow+\infty$. Hence we conclude that $\int_{0}^{T} \int_{\Omega} z(x, t) \tilde{v}(x, t) d x d t=0$.

We extend the definition of $u(x, t)$, for $x \in \Omega$ and $t \leq 0$, by $u(x, t)=U$. For all $x \in \Omega, t \in(0, T)$ and $k>0$, we have $\int_{t-k}^{t}(U-\tilde{u}(x, \tau)) d \tau \leq \int_{0}^{t}(U-\tilde{u}(x, \tau)) d \tau$. Hence we have $\int_{0}^{T} \int_{\Omega}\left[\frac{1}{k} \int_{t-k}^{t}(U-\tilde{u}(x, \tau)) d \tau\right] \tilde{v}(x, t) d x d t=0$. Using Lebesgue dominated convergence theorem, we deduce that $\int_{0}^{T} \int_{\Omega}(U-\tilde{u}(x, t)) \tilde{v}(x, t) d x d t=0$, which implies $v(x, t) .(U-u(x, t))=0$ for a.e. $(x, t) \in \Omega \times(0, T)$. This concludes the proof.

\section{Numerical example.}

In this section we give an example of application of the finite volume scheme in two space dimensions. It is applied to an actual experiment, which has been made at CEA, the 
French organism devoted to atomic energy [14].

A cylindric, fractured cement paste core is submitted to an accelerated experiment of leaching. Figure 1 shows the finite volume mesh which has been used to model a twodimensional section of the core. An implicit version of the finite volume scheme (9) and (10) has been programmed and solved using a Newton-Raphson method.

Because of the symmetry of the problem, and because the phenomenon of leaching remains local on short times, the computational area is only half of the region including the fracture (rectangle with sides equal to $1,5 \mathrm{~cm}$ and $3,5 \mathrm{~cm})$. The fracture $(2,8 \mathrm{~cm}$ long) is located at the upper part of the left side of the rectangle.

\section{Mesh with 9350 control volumes}

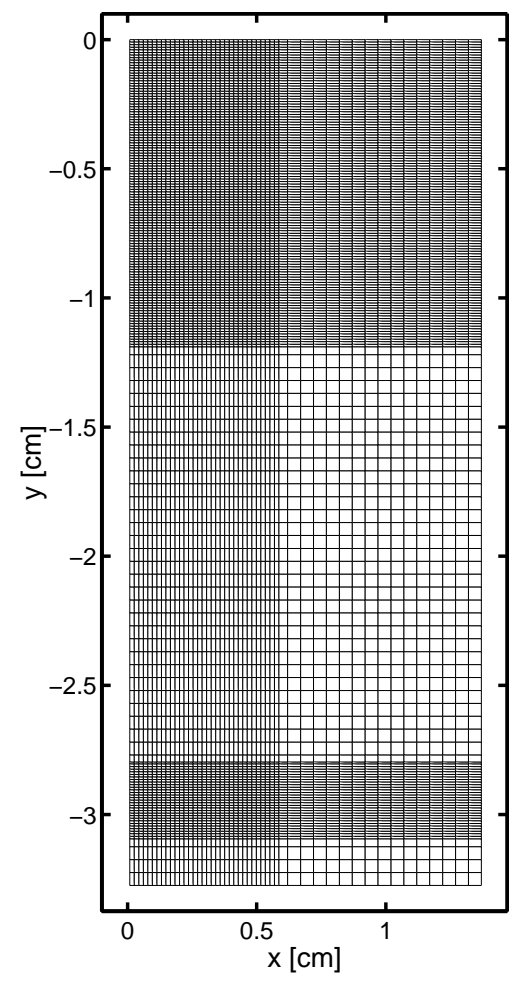

Figure 1: Finite volume mesh.

Figure 2 shows the values of $u$ after 17 days of accelerated leaching. The regularity of the solution can be seen.

Figure 3 shows the amount $v$ of solid phase after 17 days of leaching. The position of the dissolution front is fully compatible with the observed one for the boundary of the core. It is late compared to the observed one for the fracture. An explanation is that the liquid phase in which the core was introduced was continuously jerked, and therefore the diffusion process in the fracture was hidden by a non measured convective process. 


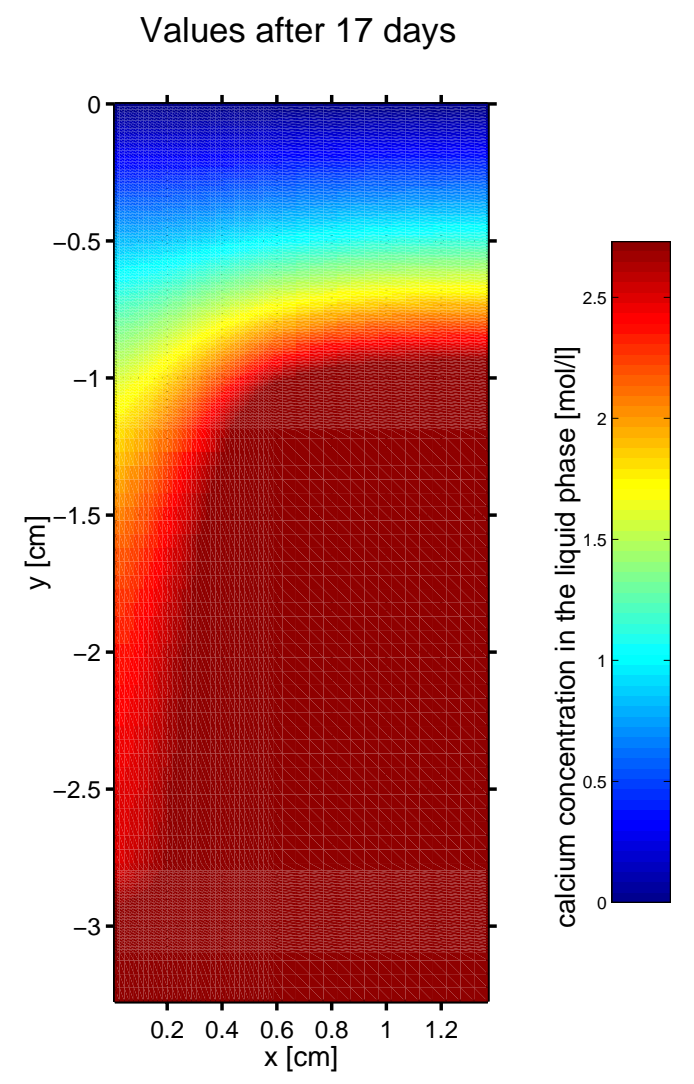

Figure 2: Concentration in the liquid phase after 17 days.

\section{References}

[1] R.A. Adams. Sobolev spaces. Academic Press, 1975.

[2] F. Adenot. Modélisation de la dégradation chimique d'une pâte de ciment, estimation dans le temps. Journées des sciences de l'ingénieur du réseau des laboratoires des ponts et Chaussées, volume II, 1994, 39-48.

[3] L.A. Baughman, N.J. Walkington. Co-volume methods for degenerate parabolic problems. Numer. Math., 64, 1993, 45-67.

[4] P. Bénilan, H. Touré. Sur l'équation générale $u_{s}=\varphi(u)_{x x}-\psi(u)_{x}+v . C$. R. Acad. Sci. Paris, Sér. I, 299, 18, 1984, 919-922.

[5] H. BRezis. Analyse fonctionnelle, théorie et applications. Collection Mathématiques appliquées pour la maîtrise, Masson, 1993.

[6] R. Dautray, J.L. Lions. Analyse mathématique et calcul numérique pour les sciences et les techniques. Collection du CEA, série scientifique Masson, Paris, 1985.

[7] R. Eymard, T. Gallouët, R. Herbin. Finite volume methods. To appear in P. Ciarlet, editor, Handbook of Numerical Analysis. 
Values after 17 days
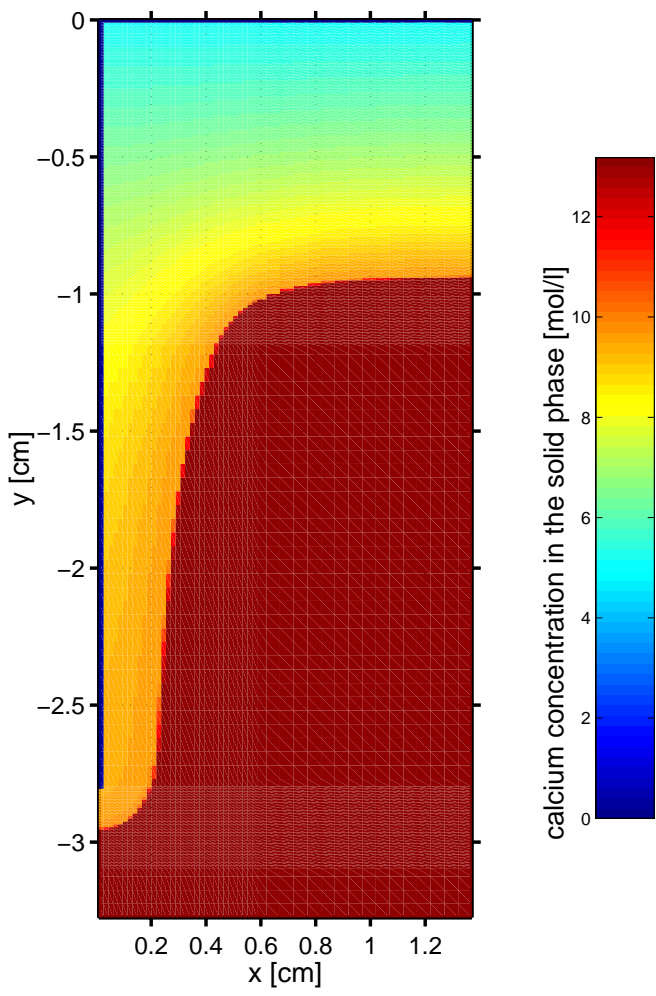

Figure 3: Concentration in the solid phase after 17 days.

[8] R. Eymard, T. Gallouët, H. Hilhorst, Y.N. Slimane. Finite volumes and nonlinear diffusion equations. Math. Mod. and Num. Anal., 1998, 32-4.

[9] M. Mainguy. Doctoral thesis. In preparation, Ecole Nationale des Ponts et Chaussées, 1999.

[10] E. Maisse, P. Moszkowicz, J. Pousin, F. Sanchez. Diffusion and dissolution in a reactive porous medium : modelling and numerical simulations. Proceedings of the International Conference On Porous Media, Saint-Etienne, France, May 22-26, 1995.

[11] P. Moszkowicz, J. Pousin, F. Sanchez., Diffusion and dissolution in a reactive porous medium. Mathematical modelling, Computational Methods For Transport in porous Media, Proceedings of Journées numériques de Besançon, Edited by J.M. Crolet, September 27-28, 1994.

[12] M.A. Peletier. Problems in degenerate diffusion. Thesis.

[13] W.H. Press, S.A. Teukolsky, W.T. Vetterling, B.P. Flannery. Numerical Recipes in C. Cambridge University Press.

[14] J.M. Torrenti, M. Mainguy, F. Adenot, C. Tognazzi. Modelling of leaching in concrete. Proceedings of EUROC-98, 1998. 
Ecole Nationale des Ponts et Chaussées, Champs-sur-Marne, France

Université de Provence, Marseille, France

Université Paris-Sud, Orsay, France

Laboratoire Central desPonts et Chaussées, Paris, France

eymard@enpc.fr 